\title{
Floral phenology and pollen potential of honey bee plants in North- East dry land areas of Amhara region, Ethiopia
}

\author{
Abebe Jenberie Wubie ${ }^{1,3, *}$, Amssalu Bezabeh ${ }^{2}$, Kefelegn Kebede ${ }^{3}$ \\ ${ }^{I}$ Sekota Dry land Agricultural Research Center, 62, Sekota, Ethiopia \\ ${ }^{2}$ Holleta Bee Research Center, Holleta, Ethiopia \\ ${ }^{3}$ Mekelle University, Mekelle, Ethiopia \\ *Abebe Jenberie Wubie is for whom correspondence E-mail:ajenberie@gmail.com
}

\begin{abstract}
Beekeeping serves as a source of additional cash income for hundreds of thousands of farmer beekeepers in the country and plays a significant role in conserving the natural resources and contributes to the globe through environmental protection. Furthermore, even if the potential and success in beekeeping development is dependent first and foremost on the type and quantity of flora available, botanical composition of natural vegetation varies significantly. This study was conducted targeting identification and documentation of major honeybee floras, their phenology and pollen potential in the study area. For this, three representative agroecologies, respective sites and localities were selected using stratified random sampling procedures. A total of 120 Beekeepers were also purposively selected and interviewed to collect relevant information. Density and frequency of flowering plants was determined using appropriate vegetation determination techniques. Pollen was collected using pollen traps and was analyzed and verified for its traceability using appropriate mellissopalynological procedures and references. Trapped pollen analysis data were also traced back to plant species level. The study has revealed that the families diversity, directly attributed to the species diversity, in sample plots were generally higher in higher altitudes of the study area and deceasing towards lower altitudes. The density of the plant species per plot were higher for herbaceous plant species represented by plant families Pedaliaceae, Asteraceae and Papilionaceae. As a tip, $73.33 \%$ of the flowering plants identified from the trapped pollen were found to be propagated by their seeds indicating the point of entrance for rehabilitation. the highest amount of pollen was collected from those plants categorized as very good in their use to quality honey production (46.23\%), in their acceptance by the bees $(56.62 \%)$ and in their abundance $(48.4 \%)$ comprising $22.22 \%$, 26.67\%, $31.11 \%$ and $28.89 \%$ of the total honey source plants identified throughout the study period respectively. Moreover, a total of $418.45 \mathrm{gm}$ of pollen $(36.11 \%, 39.08 \%$ and $20.98 \%$ from herbaceous, shrub and tree species respectively) was collected by honeybees during the study period. It was found that 12 plant species (contributing $69.24 \%$ of the pollen) have been confirmed to be major pollen source plants of the area. Generally, we would like to recommend that wise use and rehabilitation of these potentially identified bee plant species shall be considered in attempting beekeeping development and environmental protection.
\end{abstract}

Key words: Bee plants, Phenology, Bee pollen, Trapped pollen, Honey bees, Pollen source, Mellissopalynology, Dry land Ethiopia.

\section{Introduction}

Apiculture plays a significant role in the national economy of the country. It serves as a source of additional cash income for hundreds of thousands of farmer beekeepers in the country. Beekeeping plays a significant role in conserving the natural resources and contributes to the globe through environmental protection. Like all livestock species, bees require feeds for their production \& reproduction (i.e. for colony size growth) (Fichtl R. and Admasu A., 1994). The honeybee plants provide pollen and nectar as main food sources for honeybees, while, flowering plants depend on bees as pollen vectors for their sexual reproduction and this interaction is particularly important in tropical ecosystems (Admasu et al., 1999; Araújo P.A., 2005). Bees usually forage mainly on pollen and nectar (being the later is major component in honey making).

The botanical composition of natural vegetation varies depending on the topography, climate and soil type. The potential for different hive products and success in beekeeping development is dependent first and foremost on the type and quantity of flora available (Amssalu B., 1999, 2004, 2007; Segeren P., 2004). Generally, because of the diversity of plant habitat and environmental conditions and distributions, flowering season vary from place to place (Tilahun A., 2003; Amha S., 2003). However, the study area, is the area in which the regional government has given great attention to boost beekeeping productivity, has suffered frequently from sever degradation, deforestation and 
Floral phenology and pollen potential of honey bee plants in North-East dry land areas of Amhara...

low amount of rainfall and its erratic nature each year (Tilahun A., 2003) Which is much degraded and caused significant losses in vegetation cover and then number of honeybee colonies. This in turn resulted in shortage of bee forage plants to support the existing bee colony in the area (ILRI, 2000; Lakew et al., 2000). Therefore absconding and death of large number of honeybee colonies are common phenomena in the study area. This also has been amplified by the extensive loss of more than 6,000 honeybee colonies in 5 years (SWoA, 2006).

The presence of honey plants that provide pollen and nectar is very important for the existence, colony strength, production and productivity of the honeybee colony. Floral calendar of an area for beekeeping, for which it is dependent on, is a timetable that indicates the approximate durations of the blossoming (flowering) period of important bee plants and various information of the apiary (such as decision of different beekeeping managerial activities) (Amssalu B., 2004; Admasu et al., 2004). Phenology is the scientific study of periodic biological phenomena, such as the flowering of plants, in relation to climatic conditions, such as the number of days in flowering (length of flowering) (Amssalu B., 2007; FAO, 1987). Melissopalynological studies helps us to know the abundance, distribution, honey potential and phenological calendars of the vegetation in the area, when if combined beekeeping with plantations for producing high quality and quantity honey (Musarrat G., 2002). The analysis of honeybee pollen can further improve our knowledge of bee flora and vegetation.

Thus, identification and registration of honeybee flora in different agro-ecological zones, the value of different honey plants as sources of nectar and pollen or both, their life form, possible ways of propagation and their potential for honey production is a paramount importance (EARO, 2001). Moreover, beekeepers must have a working knowledge of flowering periods of both major and minor nectar and pollen producing plants in the vicinity of their apiaries for successful honey production to enable them determining when to carry out various management practices with their colonies (Francis G.S., 1990; Pearson W.D. and Braiden V., 1990).

\section{Description of the study area}

\section{Material and methods}

The study area (Fig. 1) represents three major and three sub agro-ecological zones ( hot to warm sub-moist agro ecology having an altitude of $<1500 \mathrm{~m}$, moderate or tepid sub-moist agro ecology having an altitude of 1500 $1800 \mathrm{~m}$ and cold sub-moist agro ecology of $1800-2200 \mathrm{~m}$ altitude (MoA, 1998)). The majority $(71.7 \%)$ of the area lies in the moderate sub-moist agro ecology while hot to warm sub moist comprises about $27.1 \%$ and cold submoist agro ecology occupies only $1.2 \%$ of the area (MOA, 1998). The annual rainfall varies between 350 and 650 mm (AMAREW, 2006). Generally, the topography of the study area is rugged and chain of mountain terrains which limits seriously access to the various parts.

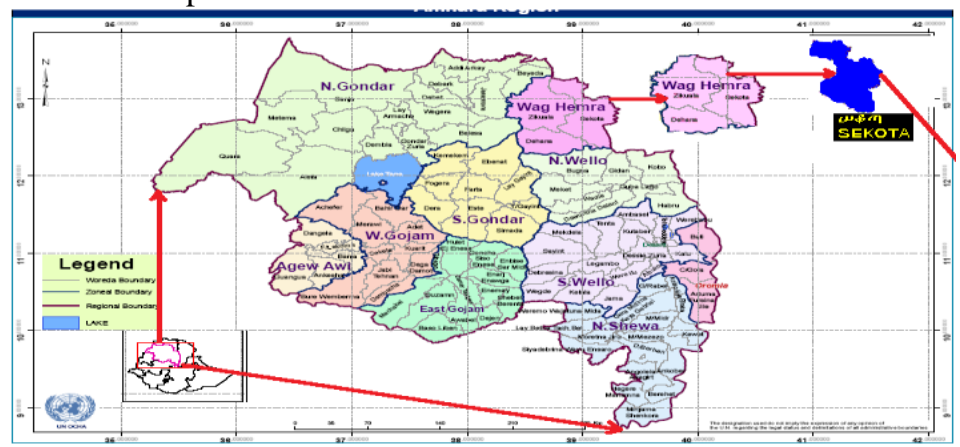

Figure 1: Administrative zones, districts and the study area in North-East Amhara, Ethiopia

\section{Data collection}

\section{Bee flora inventory}

Reconnaissance survey was employed to become familiar with the area, to get an insight on the vegetation distribution in the landscape, to observe and locate the possible traverses during the actual study. The study was conducted in three representative agro-ecologies of the study area representing lowland, which is <1500 masl, the midland, which is between 1500 and 1900 and highland, which is b/n 1900 and 2200 masl. With this, stratified random sampling procedure was followed to select the representative sites based on the strata made prior to the survey (i.e. agro-ecological representations) to exploit the different ecologies of the study area. Beekeeper farmers were also purposefully selected in order to collect the appropriate information on honey source plant lists, phenology (flowering period and duration), etc. 
Informal survey tools \& structured questionnaire were also used to strengthen the data. A total of three localities and key informants were selected for local names identification of plants. Samples of matrix ranking based prioritized flora types during formal and informal surveys were collected for further analysis according to the National Herbarium Specimen Collection techniques. Plants, which were not identified, for their taxonomic names, in the field using the indigenous knowledge and other references were categorized as unidentified. The importance of each plant in beekeeping as minor, medium and major honey sources were also determined using the PRA ranking tools and laboratory evidences. Besides, pictures of bee plants were taken to use as an identification tool and documentation.

\section{Honey plant density and frequency}

The frequency and abundance of honey source plants were estimated using a $20 \mathrm{~m} \times 20 \mathrm{~m}, 5 \mathrm{~m} \times 5 \mathrm{~m}, 1 \mathrm{~m}$ $\mathrm{x} 1 \mathrm{~m}$ quadrant for shrub and tree, herb and grass species respectively in a $2 \mathrm{~km}$ radius every $0.5 \mathrm{~km}$ distance from the hives, to understand forgeable area of honey plants by the representative bee colonies. The types of plants which were found in the quadrants were registered for their total number and local names. A total of 16 plots were recorded for each of the representative sites and the occurrence of each plant species in each of the plots was noted and density was calculated in hectare.

\section{Pollen trapping and analysis}

Six medium sized honeybee colonies were placed in each representative site of both agro-ecologies. Pollen loads, from in coming foragers, were trapped using pollen traps, fitted on the hive entrance. Trapped pollen pellets were then collected and labelled on daily basis for a total of 240 days and allowed to dry overnight at room temperature. Collected pollen pellets were also weighed for fresh and dry weight and sorted by colour. Each colour fractions were weighed independently and recorded.

Pollen grain samples from each colour representative pellets were taken and dissolved in a drop of water and mounted on a microscope slide and observed through a light microscope. On the other hand, reference slides were prepared from ripened pollen grains collected from ether washed honey plants' mature flower buds to support and verify bee pollen source plant species in the study area. Pollens which we couldn't identify botanically from both analyses techniques have been categorized as "unidentified".

\section{Statistical data analysis}

Data from pollen fresh and final weight, pollen weights collected per month, per plant family, per study sites and moisture content of honey were organized, verified, analysed and interpreted using JMP 5.0 (The Statistical Discovery Software SAS Institute Package, 2002) for ANOVA and Correlation. Data from pollen trapping were traced back to plant species level with the help of reference books, pollen atlas, and prepared pollen reference slides.

\section{Honeybee flora inventory}

\section{Results and discussion}

Important flowering plants in the study area have been presented in local and scientific names with their respective families in table 1 . Most of these plant species mentioned by respondents during the survey were similar to those identified through plant inventory and density analysis (Table 2). This has indicated that both results supported each other and indigenous knowledge of the farmers is dependable.

The bees obtain their food, and raw materials for all the hive products of interest to man such as honey, wax, and propolis directly or indirectly from plants. Among many factors, availability of potential flowering plants is the main parameter for an area to be considered as potential for honey production. According to the results of this survey, the honey bee plants of the study area comprised trees, shrubs, herbs, grasses and cultivated crops. Moreover, the species with their composition and population varies widely from area to area. Recent studies have revealed that the expansion of agriculture and rapid population growth resulted in dwindling of the forest into tiny leftovers, which are found around religious compounds and certain un-accessible escarpments of the region.

Consequently, shrubs, herbs and cultivated crops have largely replaced the previous forest vegetations which remained to be only $0.7 \%$ vegetation cover in the region (Azene et al., 1993). This shows that in the region in general and the study area in particular, there is high level of natural resources degradation which demands strong conservation and rehabilitation activities.

The diversity of families as a result of species diversity was higher in the higher altitude (28 species in18 Families) compared to mid altitude ( 24 species in 17 families) and lower altitude ( 22 species in 11 families) (Tables 3 - 5). This may be attributed to low and mid lands have low rainfall, and thus suppresses the growth of different plant species. However, this 
Floral phenology and pollen potential of honey bee plants in North-East dry land areas of Amhara...

doesn't mean that areas with higher number of plant diversity are good for honey production, as honey production is more determined by plant density and abundance. As a result, the inventory result has showed that top 15 plant species of the study area which were found in $48.12 \%$ of the sample plots constituting $77.62 \%$ of the plant species counted.were considered as dominant (major) honey bee plants (Table 2).

Honey bee plant species Guizotia abyssinica, Bidens spp., Echinops Spp., Vicia faba, Cynodon dactylon, Acacia seyal, Hypoestes trifolia, Becium grandiflorum, Acacia tortolis and Ocimum bacilicum were the top 10 (more frequent) plants in most sample plots of the highland representative area where Acacia asak, Sorghum bicolor, Sesamum indicum, Acacia mellifera, Acacia tortolis, Acacia seyal, Acacia brevispica, Bidens spp., grass spp. and Grewia bicolor were the more frequent ones in the lowland while Hypoestes trifolia, Ocimum bacilicum, Acacia tortolis, Becium grandiflorum, Bidens spp., Acacia seyal, Sorghum bicolor, Echinops spp., Vicia faba and Guizotia abyssinica were the dominant ones in the midland altitude representation.

In this regard, higher plant frequencies are known to be the best indicators of adaptation to the area and local climates. For instance Mimosaceae was the most frequent family in sample quadrate due to its growing habit in degraded areas and harsh climate conditions. This result is in agreement with the findings of Amssalu B., (2001), which indicated that density value of the plant species per plots were higher for herbaceous plant species in higher altitudes represented by plant families Pedaliaceae, Asteraceae and Papilionaceae. In this study, we have found that Asteraceae, Acanthaceae and Pedaliaceae families were the plant families in higher densities in the highland, midland and lowland representing sample plots respectively (Tables 3 - 5). On the other hand tree and shrub densities were lower in the study area due to deforestation, perhaps.

Table 1: List of honey bee plants in the study area given by the respondents (1=Very good, $2=$ Good, $3=$ Poor)

\begin{tabular}{|c|c|c|c|c|c|c|c|c|c|c|c|}
\hline No. & $\begin{array}{l}\text { Local } \\
\text { Name }\end{array}$ & $\begin{array}{l}\text { Scientific } \\
\text { Name }\end{array}$ & Family Name & $\begin{array}{c}\text { Plant } \\
\text { Abundance }\end{array}$ & $\begin{array}{c}\text { Its use } \\
\text { to } \\
\text { quality } \\
\text { Honey }\end{array}$ & $\begin{array}{c}\text { Preference } \\
\text { by the } \\
\text { bees }\end{array}$ & $\begin{array}{c}\text { Life } \\
\text { Form }\end{array}$ & $\begin{array}{c}\text { Food } \\
\text { source }\end{array}$ & $\begin{array}{c}\text { Flowering } \\
\text { Time } \\
\text { (Month) }\end{array}$ & $\begin{array}{c}\text { No of } \\
\text { days } \\
\text { stayed } \\
\text { during } \\
\text { flowering }\end{array}$ & $\begin{array}{c}\text { Means of } \\
\text { Propagation }\end{array}$ \\
\hline 1 & Echileqana & $\begin{array}{c}\text { Acacia } \\
\text { brevispica }\end{array}$ & Mimosaceae & 1 & 2 & 2 & Shrub & $\mathrm{Pol} / \mathrm{Nec}$ & May & 30 & Seed \\
\hline 2 & Gumarna & $\begin{array}{c}\text { Acacia } \\
\text { mellifera }\end{array}$ & Mimosaceae & 1 & 1 & 1 & Shrub & $\mathrm{Pol} / \mathrm{Nec}$ & May & 30 & Seed \\
\hline 3 & Tsalwa & Acacia asak & Mimosaceae & 1 & 1 & 1 & Tree & $\mathrm{Pol} / \mathrm{Nec}$ & April & 60 & Seed \\
\hline 4 & Key Girar & Acacia seyal & Mimosaceae & 2 & 2 & 2 & Tree & $\mathrm{Pol} / \mathrm{Nec}$ & May & 45 & Seed \\
\hline 5 & Abiqa & $\begin{array}{l}\text { Acacia } \\
\text { tortolis }\end{array}$ & Mimosaceae & 1 & 2 & 1 & Tree & $\mathrm{Pol} / \mathrm{Nec}$ & May & 60 & Seed \\
\hline 6 & Aluma & $\begin{array}{c}\text { Achyranthus } \\
\text { spp. }\end{array}$ & Amaranthaceae & 3 & 3 & 2 & Herb & $\mathrm{Pol} / \mathrm{Nec}$ & September & 10 & Seed \\
\hline 7 & Firtata & $\begin{array}{c}\text { Adansonia } \\
\text { digitata }\end{array}$ & Bombacaceae & 3 & 3 & 3 & Tree & $\mathrm{Pol} / \mathrm{Nec}$ & June & 25 & Seed \\
\hline 8 & Chiret & Agave spp. & Agavaceae & 2 & 3 & 3 & Shrub & $\mathrm{Pol} / \mathrm{Nec}$ & September & 30 & Vegetative \\
\hline 9 & Sibqana & $\begin{array}{c}\text { Albezia } \\
\text { amara }\end{array}$ & Mimosaceae & 2 & 3 & 3 & Tree & $\mathrm{Pol} / \mathrm{Nec}$ & May & 30 & Seed \\
\hline 10 & Malqoza & $\begin{array}{l}\text { Asparagus } \\
\text { spp. }\end{array}$ & Liliaceae & 2 & 3 & 3 & Shrub & $\mathrm{Pol} / \mathrm{Nec}$ & March & 45 & Seed \\
\hline 11 & Goza & $\begin{array}{c}\text { Balanite } \\
\text { aegyptica }\end{array}$ & Balanitaceae & 1 & 2 & 2 & Tree & $\mathrm{Pol} / \mathrm{Nec}$ & April & 45 & Seed \\
\hline 12 & Mentese & $\begin{array}{c}\text { Becium } \\
\text { grandiflorum }\end{array}$ & Lamiaceae & 1 & 1 & 1 & Shrub & $\mathrm{Pol} / \mathrm{Nec}$ & July & 90 & Seed/Veg \\
\hline 13 & $\begin{array}{l}\text { Adey } \\
\text { Abeba }\end{array}$ & Bidens spp. & Asteraceae & 3 & 2 & 1 & Herb & $\mathrm{Pol} / \mathrm{Nec}$ & August & 15 & Seed \\
\hline 14 & Shisha & $\begin{array}{c}\text { Boscia } \\
\text { anguistifolia }\end{array}$ & Capparidaceae & 2 & 3 & 3 & Tree & $\mathrm{Pol} / \mathrm{Nec}$ & April & 60 & Seed \\
\hline 15 & $\begin{array}{l}\text { Gomen } \\
\text { Zer }\end{array}$ & $\begin{array}{l}\text { Brassica } \\
\text { spp. }\end{array}$ & Brassicaceae & 2 & 3 & 2 & Herb & $\mathrm{Pol} / \mathrm{Nec}$ & September & 15 & Seed \\
\hline 16 & Wanza & $\begin{array}{l}\text { Cordia } \\
\text { africana }\end{array}$ & Boraginaceae & 3 & 2 & 1 & Tree & $\mathrm{Pol} / \mathrm{Nec}$ & May & 60 & Seed \\
\hline 17 & Serdo & $\begin{array}{l}\text { Cynodon } \\
\text { dactylon }\end{array}$ & Poaceae & 2 & 3 & 3 & Grass & Pollen & August & 5 & Seed/Veg \\
\hline
\end{tabular}




\begin{tabular}{|c|c|c|c|c|c|c|c|c|c|c|c|}
\hline 18 & Wariat & $\begin{array}{c}\text { Digitaria } \\
\text { abyssinica }\end{array}$ & Poaceae & 2 & 3 & 3 & Grass & Pollen & August & 5 & Seed/Veg \\
\hline 19 & Kushele & $\begin{array}{c}\text { Echinops } \\
\text { spp. }\end{array}$ & Asteraceae & 1 & 1 & 1 & Shrub & Nectar & September & 25 & Seed \\
\hline 20 & Akrma & $\begin{array}{c}\text { Eluesine } \\
\text { folicofolia }\end{array}$ & Poaceae & 3 & 3 & 2 & Grass & Pollen & August & 5 & Seed/Veg \\
\hline 21 & Bahir Zaf & $\begin{array}{l}\text { Eucalyptus } \\
\text { camaldlensis }\end{array}$ & Myrtaceae & 2 & 2 & 1 & Tree & $\mathrm{Pol} / \mathrm{Nec}$ & May & 30 & Seed \\
\hline 22 & Dedeho & $\begin{array}{l}\text { Euclea } \\
\text { shimperi }\end{array}$ & Ebenaceae & 1 & 1 & 1 & Shrub & $\mathrm{Pol} / \mathrm{Nec}$ & March & 45 & Seed \\
\hline 23 & Kulqual & $\begin{array}{c}\text { Euphorbia } \\
\text { Spp. }\end{array}$ & Euphorbiaceae & 3 & 2 & 2 & Shrub & $\mathrm{Pol} / \mathrm{Nec}$ & March & 15 & Vegetative \\
\hline 24 & Saha & $\begin{array}{l}\text { Grewia } \\
\text { bicolor }\end{array}$ & Tiliaceae & 3 & 2 & 2 & Shrub & $\mathrm{Pol} / \mathrm{Nec}$ & June & 21 & Seed \\
\hline 25 & Matta & $\begin{array}{l}\text { Grewia } \\
\text { villosa }\end{array}$ & Tiliaceae & 2 & 2 & 1 & Shrub & $\mathrm{Pol} / \mathrm{Nec}$ & July & 15 & Seed \\
\hline 26 & Nug & $\begin{array}{l}\text { Guizotia } \\
\text { abyssinica }\end{array}$ & Asteraceae & 2 & 2 & 1 & Herb & $\mathrm{Pol} / \mathrm{Nec}$ & September & 15 & Seed \\
\hline 27 & $\begin{array}{l}\text { Yeferenj } \\
\text { Suf }\end{array}$ & $\begin{array}{c}\text { Helianthus } \\
\text { annuus }\end{array}$ & Asteraceae & 2 & 2 & 3 & Herb & $\mathrm{Pol} / \mathrm{Nec}$ & September & 15 & Seed \\
\hline 28 & Senbelet & $\begin{array}{c}\text { Hyparrhenia } \\
\text { rufa }\end{array}$ & Poaceae & 2 & 3 & 3 & Grass & Pollen & August & 5 & Seed/Veg \\
\hline 29 & $\begin{array}{c}\text { Tej } \\
\text { Matebia }\end{array}$ & $\begin{array}{l}\text { Hypoestes } \\
\text { trifolia }\end{array}$ & Acanthaceae & 1 & 1 & 1 & Herb & $\mathrm{Pol} / \mathrm{Nec}$ & September & 15 & Seed \\
\hline 30 & Mango & $\begin{array}{l}\text { Mangifera } \\
\text { indica }\end{array}$ & Anacardiaceae & 3 & 3 & 3 & Tree & $\mathrm{Pol} / \mathrm{Nec}$ & April & 25 & Seed \\
\hline 31 & $\begin{array}{c}\text { Aba } \\
\text { Timara }\end{array}$ & $\begin{array}{c}\text { Ocimum } \\
\text { bacilicum }\end{array}$ & Lamiaceae & 1 & 1 & 1 & Herb & $\mathrm{Pol} / \mathrm{Nec}$ & August & 21 & Seed \\
\hline 32 & $\begin{array}{l}\text { Bahir } \\
\text { Kulqual }\end{array}$ & Opuntia Spp. & Cactaceae & 3 & 2 & 2 & Shrub & $\mathrm{Pol} / \mathrm{Nec}$ & June & 45 & Vegetative \\
\hline 33 & Zeyitun & $\begin{array}{l}\text { Psidium } \\
\text { guijava }\end{array}$ & Myrtaceae & 3 & 3 & 3 & Tree & $\mathrm{Pol} / \mathrm{Nec}$ & March & 30 & Seed \\
\hline 34 & Kentafa & $\begin{array}{c}\text { Pterolobium } \\
\text { stellatum }\end{array}$ & Caesalpiniaceae & 2 & 3 & 1 & Shrub & $\mathrm{Pol} / \mathrm{Nec}$ & March & 30 & Seed \\
\hline 35 & Enbacho & $\begin{array}{l}\text { Rumex } \\
\text { nervosus }\end{array}$ & Polygonaceae & 3 & 3 & 3 & Shrub & $\mathrm{Pol} / \mathrm{Nec}$ & March & 30 & Seed \\
\hline 36 & $\begin{array}{l}\text { Qundo } \\
\text { Berberie }\end{array}$ & $\begin{array}{l}\text { Schinus } \\
\text { molle }\end{array}$ & Anacardiaceae & 3 & 3 & 3 & Tree & $\mathrm{Pol} / \mathrm{Nec}$ & March & 45 & Seed \\
\hline 37 & Selit & $\begin{array}{l}\text { Sesamum } \\
\text { indicum }\end{array}$ & Pedaliaceae & 1 & 1 & 2 & Herb & $\mathrm{Pol} / \mathrm{Nec}$ & August & 15 & Seed \\
\hline 38 & Mashila & $\begin{array}{l}\text { Sorghum } \\
\text { bicolor }\end{array}$ & Poaceae & 1 & 1 & 1 & Herb & Pollen & September & 21 & Seed \\
\hline 39 & Dokima & $\begin{array}{l}\text { Syzygium } \\
\text { guineens }\end{array}$ & Myrtaceae & 3 & 3 & 3 & Tree & $\mathrm{Pol} / \mathrm{Nec}$ & April & 60 & Seed \\
\hline 40 & Ekima & $\begin{array}{l}\text { Terminalia } \\
\text { glaucescens }\end{array}$ & Combretaceae & 3 & 1 & 2 & Tree & $\mathrm{Pol} / \mathrm{Nec}$ & April & 15 & Seed \\
\hline 41 & Maget & $\begin{array}{l}\text { Trifolium } \\
\text { Spp. }\end{array}$ & Papilionaceae & 2 & 3 & 2 & Herb & Nectar & August & 5 & Seed/Veg \\
\hline 42 & Sar & $\begin{array}{l}\text { Unidentified } \\
\text { grass spp. }\end{array}$ & Poaceae & 2 & 3 & 3 & Grass & Pollen & August & 3 & Seed/Veg \\
\hline 43 & $\begin{array}{l}\text { Tihuan } \\
\text { Tila }\end{array}$ & $\begin{array}{l}\text { Verbena } \\
\text { officinalis }\end{array}$ & Verbenaceae & 3 & 3 & 3 & Herb & $\mathrm{Pol} / \mathrm{Nec}$ & July & 30 & Seed \\
\hline 44 & Girawa & $\begin{array}{l}\text { Vernonia } \\
\text { Spp. }\end{array}$ & Asteraceae & 3 & 3 & 2 & Shrub & Nectar & April & 15 & Seed \\
\hline 45 & Bakiela & Vicia faba & Papilionaceae & 1 & 2 & 2 & Herb & $\mathrm{Pol} / \mathrm{Nec}$ & September & 15 & Seed \\
\hline 46 & Bekolo & Zea mays & Poaceae & 3 & 3 & 3 & Herb & Pollen & August & 5 & Seed \\
\hline 47 & Giba & $\begin{array}{c}\text { Ziziphus } \\
\text { spinachristi }\end{array}$ & Rhamnaceae & 2 & 2 & 2 & Tree & $\mathrm{Pol} / \mathrm{Nec}$ & September & 45 & Seed \\
\hline
\end{tabular}


Floral phenology and pollen potential of honey bee plants in North-East dry land areas of Amhara...

Table 2: Density and frequency of honey plants in sample quadrants

\begin{tabular}{|c|c|c|c|c|c|}
\hline No. & Scientific Names & Family Names & $\begin{array}{l}\text { Plant } \\
\text { Count }\end{array}$ & $\begin{array}{c}\text { Plant Density Per } \\
\text { ha }\end{array}$ & $\begin{array}{c}\text { No. of Plots of } \\
\text { Observation }\end{array}$ \\
\hline 1 & Guizotia abyssinica & Asteraceae & 139 & 3475 & 1 \\
\hline 2 & Bidens spp. & Asteraceae & 117 & 2925 & 11 \\
\hline 3 & Acacia tortolis & Mimosaceae & 84 & 2100 & 8 \\
\hline 4 & Becium grandiflorum & Lamiaceae & 82 & 2050 & 5 \\
\hline 5 & Hypoestes trifolia & Acanthaceae & 78 & 1950 & 7 \\
\hline 6 & Vicia faba & Papilionaceae & 77 & 1925 & 6 \\
\hline 7 & Sorghum bicolor & Poaceae & 75 & 1875 & 13 \\
\hline 8 & Acacia asak & Mimosaceae & 73 & 1825 & 11 \\
\hline 9 & Vernonia Spp. & Asteraceae & 70 & 1750 & 2 \\
\hline 10 & Acacia mellifera & Mimosaceae & 68 & 1700 & 6 \\
\hline 11 & Sesamum indicum & Pedaliaceae & 66 & 1650 & 10 \\
\hline 12 & Acacia seyal & Mimosaceae & 65 & 1625 & 13 \\
\hline 13 & Ocimum bacilicum & Lamiaceae & 64 & 1600 & 13 \\
\hline 14 & Brassica spp. & Brassicaceae & 63 & 1575 & 2 \\
\hline 15 & Echinops spp. & Asteraceae & 61 & 1525 & 7 \\
\hline 16 & Un-Identified grass spp. & Poaceae & 60 & 1500 & 5 \\
\hline 17 & Acacia brevispica & Mimosaceae & 59 & 1475 & 6 \\
\hline 18 & Albezia amara & Mimosaceae & 58 & 1450 & 5 \\
\hline 19 & Hyparrhenia rufa & Poaceae & 55 & 1375 & 8 \\
\hline 20 & Grewia bicolor & Tiliaceae & 52 & 1300 & 3 \\
\hline 29 & Euclea shimperi & Ebenaceae & 52 & 1300 & 7 \\
\hline 21 & Cyperus spp. & Cyperaceae & 51 & 1275 & 5 \\
\hline 22 & Eluesine floccifolia & Poaceae & 49 & 1225 & 6 \\
\hline 30 & Aloe berhana & Liliaceae & 48 & 1200 & 4 \\
\hline 24 & Opuntia Spp. & Cactaceae & 47 & 1175 & 6 \\
\hline 25 & Cynodon dactylon & Poaceae & 45 & 1125 & 13 \\
\hline 31 & Eucalyptus camaldlensis & Myrtaceae & 27 & 675 & 4 \\
\hline 32 & Ziziphus spinachristi & Rhamnaceae & 25 & 625 & 4 \\
\hline 27 & Rumex nervosus & Polygonaceae & 23 & 575 & 4 \\
\hline 33 & Euphorbia Spp. & Euphorbiaceae & 17 & 425 & 3 \\
\hline 36 & Balanite aegyptica & Balanitaceae & 17 & 425 & 4 \\
\hline 34 & Achyranthus spp. & Amaranthaceae & 15 & 375 & 8 \\
\hline 40 & Pterolobium stellatum & Caesalpiniaceae & 13 & 325 & 1 \\
\hline 35 & Agave sisalana & Agavaceae & 9 & 225 & 5 \\
\hline 23 & Cordia africana & Boraginaceae & 8 & 200 & 1 \\
\hline 26 & Helianthus annus & Asteraceae & 7 & 175 & 1 \\
\hline 28 & Digitaria abyssinica & Poaceae & 5 & 125 & 1 \\
\hline 37 & Grewia villosa & Tiliaceae & 5 & 125 & 4 \\
\hline 43 & Asparagus spp. & Liliaceae & 5 & 125 & 6 \\
\hline 38 & Boscia anguistifolia & Capparidaceae & 4 & 100 & 4 \\
\hline 41 & Terminalia glaucescens & Combretaceae & 4 & 100 & 1 \\
\hline 42 & Carica papaya & Caricaceae & 3 & 75 & 3 \\
\hline 39 & Cadaba farinosa & Capparidaceae & 2 & 50 & 2 \\
\hline
\end{tabular}

Table 3: Honey plant families and their frequency of occurrence in the highlands

\begin{tabular}{cccc}
\hline Family Name & Plant Count & Plant Density per ha & No. of Species \\
\hline Asteraceae & 504 & 700.0 & 5 \\
Papilionaceae & 100 & 416.7 & 2 \\
Lamiaceae & 149 & 206.9 & 1 \\
Acanthaceae & 44 & 157.1 & 2 \\
Mimosaceae & 114 & 135.7 & 5 \\
Poaceae & 198 & 130.3 & 1 \\
Tiliaceae & 14 & 116.7 & 1 \\
Cyperaceae & 20 & 100.0 & 1 \\
Polygonaceae & 16 & 100.0 & 1 \\
Ebenaceae & 27 & 96.4 & 1 \\
Brassicaceae & 17 & 85.0 & 1 \\
Cactaceae & 17 & 70.8 & 1 \\
Agavaceae & 9 & 45.0 & 1 \\
Boraginaceae & 7 & 43.8 & 1 \\
Euphorbiaceae & 4 & 33.3 & 1 \\
Myrtaceae & 5 & 31.3 & 1 \\
Caesalpiniaceae & 1 & 25.0 & 1 \\
Caricaceae & 2 & 16.7 & \\
\hline
\end{tabular}


Floral phenology and pollen potential of honey bee plants in North-East dry land areas of Amhara...

Table 4: Honey plant families and their frequency of occurrence in the midlands

\begin{tabular}{cccc}
\hline Family Name & Plant Count & $\begin{array}{c}\text { Plant Density per } \\
\text { ha }\end{array}$ & No. of Species \\
\hline Acanthaceae & 113 & 256.8 & 1 \\
Cyperaceae & 24 & 200.0 & 1 \\
Lamiaceae & 152 & 181.0 & 2 \\
Mimosaceae & 132 & 165.0 & 2 \\
Papilionaceae & 32 & 160.0 & 1 \\
Asteraceae & 132 & 137.5 & 4 \\
Brassicaceae & 9 & 112.5 & 1 \\
Polygonaceae & 7 & 87.5 & 1 \\
Poaceae & 104 & 81.3 & 4 \\
Cactaceae & 16 & 80.0 & 1 \\
Myrtaceae & 10 & 41.7 & 1 \\
Boraginaceae & 1 & 25.0 & 1 \\
Combretaceae & 1 & 25.0 & 1 \\
Ebenaceae & 2 & 25.0 & 1 \\
Euphorbiaceae & 3 & 25.0 & 1 \\
Liliaceae & 6 & 25.0 & \\
\hline
\end{tabular}

Table 5: Honey plant families and their frequency of occurrence in the lowlands

\begin{tabular}{cccc}
\hline Family Name & Plant Count & $\begin{array}{c}\text { Plant Density per } \\
\text { ha }\end{array}$ & No. of Species \\
\hline Pedaliaceae & 160 & 400.0 & 1 \\
Mimosaceae & 591 & 378.8 & 6 \\
Poaceae & 224 & 350.0 & 3 \\
Asteraceae & 69 & 123.2 & 2 \\
Rhamnaceae & 15 & 93.8 & 1 \\
Cactaceae & 11 & 91.7 & 1 \\
Tiliaceae & 21 & 87.5 & 2 \\
Amaranthaceae & 15 & 46.9 & 1 \\
Balanitaceae & 7 & 43.8 & 1 \\
Liliaceae & 13 & 32.5 & 2 \\
Capparidaceae & 6 & 25.0 & 2 \\
\hline
\end{tabular}

\section{Honey plant phenology (flowering period)}

The phenological picture of total flowering, defines the changes in the seasonal landscape over the study area (Figure 2). Honey plants flower throughout the study period except in June, July and October in midland, highland and lowland representative sites respectively. However, the highest proportion of honey plants flowers during August through October, with the peak in August and September and March to May with their peak in March (Figure 2). This has explained that August to October is the major and March to May is the minor honey flow periods of the study area. This may explain the reason why flowering period differ also in each agro-ecologies of the study area. Thus honey plant phenology in the study area is considered to be biphasic. However, the minor honey flow period of the area is preceded by the short rainy season ( 'Belg'), mostly which is not frequent.

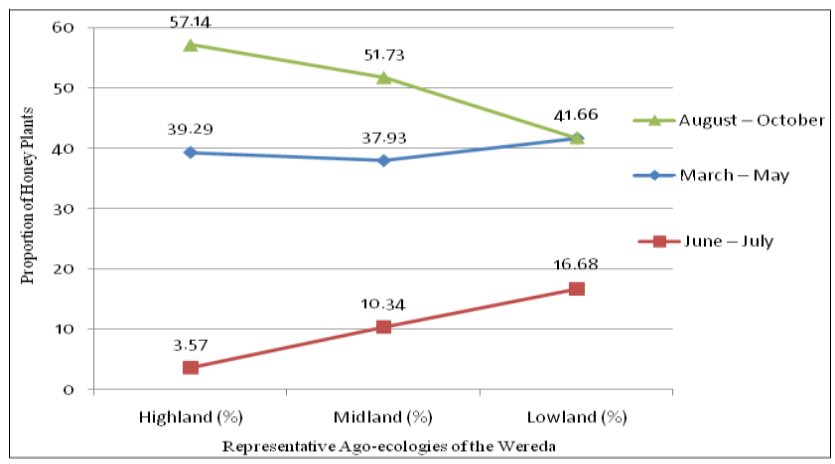

Figure 2: Flowering periods of honey plants

As it is explained by Hepburn \& Radloff (1998) and we understood, the major rains occur in summer ('Kiremt') following which flowering intensity reaches a peak in autumn, flowering phenology characteristic of the herbaceous flora throughout the country at large and the study area in particular is highly boosted. 
Floral phenology and pollen potential of honey bee plants in North-East dry land areas of Amhara...

\section{Trapped pollen analysis}

From the trapped pollen analysis, we have found that pollens of 27 plant families comprised of 45 species of plants were collected by honey bees of which $8.89 \%, 31.11 \%, 35.56 \%$ and $24.44 \%$ were grasses, herbs, shrubs and trees respectively (Table 6). In this study, the honey bees have collected pollen for a total of 207 days in the highland, 193 days in the midland and 196 days in the lowland areas.

Table 6: Nature of honey plants

\begin{tabular}{ccccccccccc}
\hline Site & $\begin{array}{c}\text { No. of Plant } \\
\text { Species }\end{array}$ & $\begin{array}{c}\text { No. of } \\
\text { Families }\end{array}$ & Grasses & \% & Herbs & \% & Shrubs & \% & Trees & \% \\
\hline Highland & 28 & 16 & 2 & 7.1 & 8 & 28.6 & 10 & 35.7 & 8 & 28.6 \\
Midland & 29 & 17 & 3 & 10.3 & 9 & 31.0 & 10 & 34.5 & 7 & 24.1 \\
Lowland & 24 & 15 & 1 & 4.2 & 6 & 25.0 & 7 & 29.2 & 10 & 41.7 \\
\hline
\end{tabular}

As suggested by Diaz L. et al., (1998) from a study conducted in Italy that pollen composition from beehives showed the different characteristics of local vegetations. This also showed that the local floras have characteristic plant associations that are reflected in the corresponding spectrum of pollen types represented in the local honeys and honey carries its certificates of origin (Nuru A. et at., 2001; Nuru A., 2007).

When we see the food sources obtained by honeybees from bee plants of the area, all grass species were only pollen source plants. Whereas, among a total of 14 herb species, 12 of them provided both pollen and nectar and 1 species is a nectar source and the remaining 1 is a pollen source plant. 14 species out of 16 shrubs provided pollen and nectar and only 2 are nectar sources. However all tree species identified was found to be both a pollen and nectar source plants (Table 7).

Table 7: Food sources of honey plants

\begin{tabular}{ccccccccc}
\hline Site & $\begin{array}{c}\text { No. of Plant } \\
\text { Species }\end{array}$ & $\begin{array}{c}\text { No. of } \\
\text { Families }\end{array}$ & $\begin{array}{c}\text { Pollen Source } \\
\text { Plants }\end{array}$ & $\%$ & $\begin{array}{c}\text { Pollen and Nectar } \\
\text { Source Plants }\end{array}$ & $\%$ & $\begin{array}{c}\text { Nectar Source } \\
\text { Plants }\end{array}$ & $\%$ \\
\hline Highland & 28 & 16 & 0 & 0.0 & 25 & 89.3 & 3 & 10.7 \\
Midland & 29 & 17 & 1 & 3.4 & 25 & 86.2 & 3 & 10.3 \\
Lowland & 24 & 15 & 0 & 0.0 & 22 & 91.7 & 2 & 8.3 \\
\hline
\end{tabular}

The identification of bee-collected pollen loads from pollen traps indicated not only the plants from which the honeybees had collected the pollen but it also showed the relative importance of each plant species as a pollen source. The total amount of pollen collection from lower altitude representations was lower in quantity. This may be attributed to high temperature, insufficient moisture and erratic rainfall enforcing the flowering plants to bloom for short period of time while the flowering periods of the plants in the higher altitudes extends up to end of October enhancing longer period of blooming which has resulted in higher amounts of pollen collections.

Even if there are 250,000 species of plants throughout the world visited by honeybees of which 4000 are considered to be the most important honey source plants (Irene, 2005), at least 45 species of bee plants have been visited by honeybees in the study area. However, a final perspective should be gained by considering the relative breadth and depth of plant species utilization by honeybees. Although honeybees are considered as florally promiscuous, studies on pollen source plant species uniformly demonstrated that a narrow band of species in a wide spectrum of floral choices was actually used at a significant level in the study area.

As explained by various studies, the trait of flower constancy has gone a long way in explaining selectivity of honeybees in pollen foraging. Likewise, the temporal periodicity of available pollen in bee plants further modified a final account of pollen utilization over the seasons (Amssalu B., 1999).

Even though honeybees frequently are making use of the floral resources available closer to the beehives, the presence of a specific combination of pollen types in a sample has indicated their botanical and geographical origins (Diaz L. et al., 1998). Furthermore, as it has been observed in this study, this has indicated the width and breadth of floral resources in the area. However, Amssalu B. (2001) reported that even if different types of honey bee plants were observed in the highlands of Ethiopia, the broad generalizations relating honeybee colony cycles to the phenology of flowering and climate do not reflect the collection of pollen by honeybees with regard to seasonal plant species diversity.

As it was evidenced by respondents, very good, good and poor bee plants in different parameters have showed a significant difference in pollen yield among themselves. Consequently, very good plants in their use to quality honey production, in preference by the honey bees and in abundance have provided the highest pollen yield (46.23\%, 56.62\% and $48.40 \%$ respectively) Table 8. However, the amount of pollen collected by honeybees varied over the seasons. This result has also showed that in the absence of pollen analysis, use of experienced beekeeper farmers could be worthy enough to identify the potential honey source plants in a given area. 
Floral phenology and pollen potential of honey bee plants in North-East dry land areas of Amhara...

Table 8: Criteria of selection of bee plants and their contribution to total pollen yield.

\begin{tabular}{cccccc}
\hline Criteria of Selection & Grades Given & $\begin{array}{c}\text { No. of Plant } \\
\text { Species }\end{array}$ & $\%$ & $\begin{array}{c}\text { Total Pollen } \\
\text { Yield (gm) }\end{array}$ & $\begin{array}{c}\text { \% } \\
\text { Contribution }\end{array}$ \\
\hline \multirow{2}{*}{ Its Use to Quality Honey } & Very good & 12 & 26.67 & 193.43 & 46.23 \\
Production & Good & 10 & 22.22 & 123.86 & 29.60 \\
& Poor & 23 & 51.11 & 101.16 & 24.17 \\
\hline \multirow{3}{*}{ Preference by the bees } & Very good & 14 & 31.11 & 236.93 & 56.62 \\
& Good & 18 & 40.00 & 136.44 & 32.61 \\
& Poor & 13 & 28.89 & 45.08 & 10.77 \\
\hline \multirow{3}{*}{ Plant Abundance } & Very good & 13 & 28.89 & 202.52 & 48.40 \\
& Good & 15 & 33.33 & 120.94 & 28.90 \\
& Poor & 17 & 37.78 & 94.99 & 22.70 \\
\hline
\end{tabular}

Furthermore, the respondents have indicated that $73.33 \%$ of the flowering plants identified from the trapped pollen are propagated by their seeds only, $15.56 \%$ by their seeds and vegetative means, and $8.89 \%$ by vegetative means only (Table 9 ).

Table 9: Methods of propagation of bee plants

\begin{tabular}{ccccccccc}
\hline Site & $\begin{array}{c}\text { No. of Plant } \\
\text { Species }\end{array}$ & $\begin{array}{c}\text { No. of } \\
\text { Families }\end{array}$ & Seed & $\%$ & Seed/Vegetative & \% & Vegetative & $\%$ \\
\hline Highland & 28 & 16 & 21 & 75.0 & 4 & 14.3 & 3 & 10.7 \\
Midland & 29 & 17 & 21 & 72.4 & 4 & 13.8 & 4 & 13.8 \\
Lowland & 24 & 15 & 20 & 83.3 & 1 & 4.2 & 3 & 12.5 \\
\hline
\end{tabular}

Major pollen source plant species identified in the trapped pollen analysis were varied across sampling areas of the study area which has confirmed that the type of vegetation reflects the agro-ecologies of the area. In this study, a higher quantity of pollen $(183.43 \mathrm{gm})$ was collected from the highland representations than the midland $(145.92 \mathrm{gm})$ and lowland $(91.22 \mathrm{gm})$ areas (Tables $10-12)$. More specifically, $65.94 \%, 67.52 \%$ and $56.44 \%$ of the total pollen collected was from 7,8 and 10 plant species in the mid, lower and higher altitudes of the study area respectively. This has implied that species diversity of honey bee plants was higher in the highland sites. In fact, this is attributed to the higher moisture in the highland has favoured the growth and flowering of various bee plants compared to the lowland and midland representatives. Meanwhile, Acacia tortolis (7.74\%), Ocimum bacilicum (6.99\%), Becium grandiflorum (6.45\%), Hypoestes trifolia (6.44\%), Sorghum bicolor (6.10\%), Bidens spp. (5.85\%) and Guizotia abyssinica (5.69\%) were the major contributors (about $45.26 \%$ ) of pollen income in the study area (Table 13). Even though honeybees collected pollen from 27 plant families in this study, the major pollen source bee plant families were Asteraceae, Mimosaceae, Lamiaceae, Poaceae, and Acanthaceae contributing 19.95\%, 15.19\%, $14.33 \%, 9.74 \%$ and $7.24 \%$ of the total pollen collection respectively (Table 14 ).

Table 10: Identified bee plant species from trapped pollen in the midland

\begin{tabular}{|c|c|c|c|c|c|c|c|c|}
\hline Local Name & Scientific Name & Family Name & $\begin{array}{c}\text { Pollen } \\
\text { Weight } \\
\text { (gm) }\end{array}$ & $\begin{array}{l}\text { Life } \\
\text { Form }\end{array}$ & $\begin{array}{c}\text { Food } \\
\text { source }\end{array}$ & $\begin{array}{c}\text { Flowering } \\
\text { Time (Month) }\end{array}$ & $\begin{array}{c}\text { No of days } \\
\text { stayed } \\
\text { during } \\
\text { flowering } \\
\end{array}$ & $\begin{array}{c}\text { Means of } \\
\text { Propagatio } \\
\mathbf{n}\end{array}$ \\
\hline Tej Matebia & Fypoestes trifolia & Acanthaceae & 18.32 & Herb & $\mathrm{Pol} / \mathrm{Nec}$ & September & 15 & Seed \\
\hline Mentese & Becium grandiflorum & Lamiaceae & 17.11 & Shrub & $\mathrm{Pol} / \mathrm{Nec}$ & July & 90 & Seed/Veg \\
\hline Aba Timara & Ocimum bacilicum & Lamiaceae & 15.69 & Shrub & $\mathrm{Pol} / \mathrm{Nec}$ & August & 21 & Seed \\
\hline Abiqa & Acacia tortolis & Mimosaceae & 13.89 & Tree & $\mathrm{Pol} / \mathrm{Nec}$ & May & $60-75$ & Seed \\
\hline Kushele & Echinops spp. & Asteraceae & 10.72 & Shrub & Nectar & September & 25 & Seed \\
\hline Nug & Guizotia abyssinica & Asteraceae & 10.52 & Herb & $\mathrm{Pol} / \mathrm{Nec}$ & October & 15 & Seed \\
\hline Mashila & Sorghum bicolor & Poaceae & 8.57 & Herb & $\mathrm{Pol} / \mathrm{Nec}$ & September & 21 & Seed \\
\hline Gomen Zer & Brassica spp. & Brassicaceae & 5.36 & Herb & $\mathrm{Pol} / \mathrm{Nec}$ & September & 15 & Seed \\
\hline Adey Abeba & Bidens spp. & Asteraceae & 4.85 & Herb & $\mathrm{Pol} / \mathrm{Nec}$ & August & 10 & Seed \\
\hline Sar & Un Identified spp. & Poaceae & 4.23 & Grass & Pollen & August & 5 & Seed/Veg \\
\hline Bahir zaf & $\begin{array}{c}\text { Eucalyptus } \\
\text { camaldulensis }\end{array}$ & Myrtaceae & 3.99 & Tree & $\mathrm{Pol} / \mathrm{Nec}$ & April & $90-120$ & Seed \\
\hline Bakiela & Vicia faba & Papilionaceae & 3.45 & Herb & $\mathrm{Pol} / \mathrm{Nec}$ & August & 25 & Seed \\
\hline Saha & Grewia bicolor & Tiliaceae & 2.93 & Shrub & $\mathrm{Pol} / \mathrm{Nec}$ & June & 21 & Seed \\
\hline Eriet & Aloe berhana & Liliaceae & 2.82 & Shrub & $\mathrm{Pol} / \mathrm{Nec}$ & September & 45 & Vegetative \\
\hline Kentafa & Pterolobium spp. & Caesalpiniaceae & 2.72 & Shrub & $\mathrm{Pol} / \mathrm{Nec}$ & September & 30 & Seed \\
\hline Giba & Ziziphus spinachristi & Rhamnaceae & 2.58 & Tree & $\mathrm{Pol} / \mathrm{Nec}$ & September & 45 & Seed \\
\hline
\end{tabular}


Floral phenology and pollen potential of honey bee plants in North-East dry land areas of Amhara...

\begin{tabular}{|c|c|c|c|c|c|c|c|c|}
\hline Girawa & Vernonia Spp. & Asteraceae & 2.45 & Shrub & Nectar & April & 15 & Seed \\
\hline Bahir Kulqual & Opuntia Spp. & Cactaceae & 2.41 & Shrub & $\mathrm{Pol} / \mathrm{Nec}$ & May & 30 & Vegetative \\
\hline Qundo Berbere & Schinus molle & Anacardiaceae & 1.90 & Tree & $\mathrm{Pol} / \mathrm{Nec}$ & March & 45 & Seed \\
\hline Maget & Trifolium Spp. & Papilionaceae & 1.62 & Herb & Nectar & July & 15 & Seed \\
\hline Tirunba Abeba & $\begin{array}{c}\text { Zantadescha } \\
\text { aethiopica }\end{array}$ & Araceae & 1.56 & Herb & $\mathrm{Pol} / \mathrm{Nec}$ & September & 15 & Seed \\
\hline Suf & Helianthus annuus & Asteraceae & 1.44 & Herb & $\mathrm{Pol} / \mathrm{Nec}$ & September & 15 & Seed \\
\hline Bekolo & Zea mays & Poaceae & 1.34 & Herb & Pollen & August & 5 & Seed \\
\hline Feto & Lepidium sativum & Brassicaceae & 1.13 & Herb & $\mathrm{Pol} / \mathrm{Nec}$ & September & 15 & Seed \\
\hline Sensel & Justicia shimeriana & Acanthaceae & 1.04 & Shrub & $\mathrm{Pol} / \mathrm{Nec}$ & August & 15 & Seed/Veg \\
\hline Kulqual & Euphorbia Spp. & Euphorbiaceae & 0.80 & Shrub & $\mathrm{Pol} / \mathrm{Nec}$ & October & 45 & Vegetative \\
\hline Gumero & Capparis micrantha & Capparidaceae & 0.36 & Shrub & $\mathrm{Pol} / \mathrm{Nec}$ & June & 25 & Seed/Veg \\
\hline
\end{tabular}

Table 11: Identified bee plant species from trapped pollen in the lowland

\begin{tabular}{|c|c|c|c|c|c|c|c|c|}
\hline Local Name & Scientific Name & Family Name & $\begin{array}{l}\text { Pollen } \\
\text { Weight } \\
\text { (gm) }\end{array}$ & $\begin{array}{l}\text { Life } \\
\text { Form }\end{array}$ & $\begin{array}{l}\text { Food } \\
\text { Source }\end{array}$ & $\begin{array}{l}\text { Flowering Time } \\
\text { (Month) }\end{array}$ & $\begin{array}{l}\text { No. of days } \\
\text { stayed } \\
\text { during } \\
\text { flowering }\end{array}$ & $\begin{array}{c}\text { Means of } \\
\text { Propagatio } \\
\text { n }\end{array}$ \\
\hline Tsalwa & Acacia asak & Mimosaceae & 10.17 & Tree & $\mathrm{Pol} / \mathrm{Nec}$ & April & 60 & Seed \\
\hline Gumarna & Acacia mellifera & Mimosaceae & 9.44 & Shrub & $\mathrm{Pol} / \mathrm{Nec}$ & May & 30 & Seed \\
\hline Key Girar & Acacia seyal & Mimosaceae & 8.05 & Tree & $\mathrm{Pol} / \mathrm{Nec}$ & May & 30 & Seed \\
\hline Abiqa & Acacia tortolis & Mimosaceae & 7.90 & Tree & $\mathrm{Pol} / \mathrm{Nec}$ & May & 60 & Seed \\
\hline Selit & Sesamum indicum & Pedaliaceae & 7.84 & Herb & $\mathrm{Pol} / \mathrm{Nec}$ & August & 15 & Seed \\
\hline Mashila & Sorghum bicolor & Poaceae & 7.42 & Herb & $\mathrm{Pol} / \mathrm{Nec}$ & September & 15 & Seed \\
\hline Adey Abeba & Bidens spp. & Asteraceae & 5.43 & Herb & $\mathrm{Pol} / \mathrm{Nec}$ & August & 15 & Seed \\
\hline Bahir Kulqual & Opuntia Spp. & Cactaceae & 5.34 & Shrub & $\mathrm{Pol} / \mathrm{Nec}$ & July & 30 & Vegetative \\
\hline Eriet & Aloe spp. & Liliaceae & 4.38 & Shrub & $\mathrm{Pol} / \mathrm{Nec}$ & September & 20 & Vegetative \\
\hline Saha & Grewia bicolor & Tiliaceae & 4.26 & Shrub & $\mathrm{Pol} / \mathrm{Nec}$ & September & 10 & Seed \\
\hline Giba & $\begin{array}{c}\text { Ziziphus } \\
\text { spinachristi }\end{array}$ & Rhamnaceae & 4.19 & Tree & $\mathrm{Pol} / \mathrm{Nec}$ & September & 45 & Seed \\
\hline Echileqana & Acacia brevispica & Mimosaceae & 3.52 & Shrub & $\mathrm{Pol} / \mathrm{Nec}$ & May & 30 & Seed \\
\hline Kulqual & Euphorbia Spp. & Euphorbiaceae & 3.12 & Shrub & $\mathrm{Pol} / \mathrm{Nec}$ & March & 15 & Vegetative \\
\hline Sar & Un-Identified spp. & Poaceae & 3.12 & Grass & Pollen & August & 3 & Seed/Veg \\
\hline Girawa & Vernonia Spp. & Asteraceae & 3.11 & Herb & Nectar & August & 25 & Seed \\
\hline Goza & Balanite aegyptica & Balanitaceae & 1.77 & Tree & $\mathrm{Pol} / \mathrm{Nec}$ & April & 45 & Seed \\
\hline Aluma & Achyranthus spp. & Amaranthaceae & 1.67 & Herb & $\mathrm{Pol} / \mathrm{Nec}$ & September & 10 & Seed \\
\hline Maget & Trifolium Spp. & Papilionaceae & 0.31 & Herb & Nectar & August & 3 & Seed \\
\hline Gorteb & Plantago spp. & Plantaginaceae & 0.18 & Grass & Pollen & August & 10 & Seed \\
\hline
\end{tabular}

Table 12: Identified bee plant species from trapped pollen in the highland

\begin{tabular}{|c|c|c|c|c|c|c|c|c|}
\hline $\begin{array}{l}\text { Local } \\
\text { Name }\end{array}$ & Scientific Name & Family Name & $\begin{array}{c}\text { Pollen } \\
\text { Weight } \\
\text { (gm) }\end{array}$ & $\begin{array}{l}\text { Life } \\
\text { Form }\end{array}$ & $\begin{array}{l}\text { Food } \\
\text { Source }\end{array}$ & $\begin{array}{c}\text { Flowering } \\
\text { Time (Month) }\end{array}$ & $\begin{array}{l}\text { No of days stayed } \\
\text { during flowering }\end{array}$ & $\begin{array}{l}\text { Means of } \\
\text { Propagation }\end{array}$ \\
\hline $\begin{array}{l}\text { Adey } \\
\text { Abeba }\end{array}$ & Bidens spp. & Asteraceae & 14.18 & Herb & $\mathrm{Pol} / \mathrm{Nec}$ & September & 15 & Seed \\
\hline Aba Timara & Ocimum bacilicum & Lamiaceae & 13.54 & Shrub & $\mathrm{Pol} / \mathrm{Nec}$ & August & 25 & Seed \\
\hline Nug & Guizotia abyssinica & Asteraceae & 13.31 & Herb & $\mathrm{Pol} / \mathrm{Nec}$ & September & 15 & Seed \\
\hline Abiqa & Acacia tortolis & Mimosaceae & 10.60 & Tree & $\mathrm{Pol} / \mathrm{Nec}$ & April & 30 & Seed \\
\hline Mentese & Becium grandiflorum & Lamiaceae & 9.86 & Shrub & $\mathrm{Pol} / \mathrm{Nec}$ & August & 60 & Seed/Veg \\
\hline Mashila & Sorghum bicolor & Poaceae & 9.54 & Herb & $\mathrm{Pol} / \mathrm{Nec}$ & September & 35 & Seed \\
\hline Girawa & Vernonia Spp. & Asteraceae & 9.25 & Shrub & Nectar & August & 15 & Seed \\
\hline $\begin{array}{c}\text { Tej } \\
\text { Matebia }\end{array}$ & Hypoestes trifolia & Acanthaceae & 8.63 & Herb & $\mathrm{Pol} / \mathrm{Nec}$ & September & 15 & Seed \\
\hline Bahir Zaf & Eucalyptus camaldlensis & Myrtaceae & 7.47 & Tree & $\mathrm{Pol} / \mathrm{Nec}$ & May & 30 & Seed \\
\hline Kushele & Echinops Spp. & Asteraceae & 7.15 & Shrub & Nectar & October & 60 & Seed \\
\hline Wanza & Cordia africana & Boraginaceae & 6.70 & Tree & $\mathrm{Pol} / \mathrm{Nec}$ & March & 21 & Seed \\
\hline Saha & Grewia bicolor & Tiliaceae & 6.62 & Shrub & $\mathrm{Pol} / \mathrm{Nec}$ & June & 21 & Seed \\
\hline
\end{tabular}


Floral phenology and pollen potential of honey bee plants in North-East dry land areas of Amhara...

\begin{tabular}{|c|c|c|c|c|c|c|c|c|}
\hline Gomen Zer & Brassica spp. & Brassicaceae & 6.29 & Herb & $\mathrm{Pol} / \mathrm{Nec}$ & September & 15 & Seed \\
\hline Kulqual & Euphorbia Spp. & Euphorbiaceae & 5.02 & Shrub & $\mathrm{Pol} / \mathrm{Nec}$ & October & 25 & Vegetative \\
\hline Kentafa & Pterolobium stellatum & Caesalpiniaceae & 4.59 & Shrub & $\mathrm{Pol} / \mathrm{Nec}$ & March & 30 & Seed \\
\hline Bakiela & Vicia faba & Papilionaceae & 4.18 & Herb & $\mathrm{Pol} / \mathrm{Nec}$ & September & 15 & Seed \\
\hline Eriet & Aloe berhana & Liliaceae & 4.05 & Shrub & $\mathrm{Pol} / \mathrm{Nec}$ & September & 15 & Vegetative \\
\hline $\begin{array}{l}\text { Yefyel } \\
\text { Gomen }\end{array}$ & Plectranthus spp. & Lamiaceae & 3.76 & Herb & $\mathrm{Pol} / \mathrm{Nec}$ & August & 15 & Seed \\
\hline Feto & Lepidium sativum & Brassicaceae & 3.52 & Herb & $\mathrm{Pol} / \mathrm{Nec}$ & September & 15 & Seed \\
\hline Akrma & Eluesine folicofolia & Poaceae & 3.38 & Grass & Pollen & August & 5 & Seed/Veg \\
\hline Giba & Ziziphus spinacristi & Rhamnaceae & 3.32 & Tree & $\mathrm{Pol} / \mathrm{Nec}$ & September & 45 & Seed \\
\hline Sar & Un-Identified spp. & Poaceae & 3.15 & Grass & Pollen & August & 5 & Seed/Veg \\
\hline Maget & Trifolium Spp. & Papilionaceae & 2.35 & Herb & Nectar & August & 5 & Seed/Veg \\
\hline Enbacho & Rumex nervosus & Polygonaceae & 2.34 & Shrub & $\mathrm{Pol} / \mathrm{Nec}$ & March & 30 & Seed \\
\hline Sensel & Justicia spp. & Acanthaceae & 2.32 & Shrub & $\mathrm{Pol} / \mathrm{Nec}$ & August & 15 & Seed/Veg \\
\hline $\begin{array}{l}\text { Tirunba } \\
\text { Abeba }\end{array}$ & Zantadescha spp. & Araceae & 2.19 & Herb & $\mathrm{Pol} / \mathrm{Nec}$ & September & 15 & Seed \\
\hline $\begin{array}{l}\text { Bahir } \\
\text { Kulqual }\end{array}$ & Opuntia Spp. & Cactaceae & 2.04 & Shrub & $\mathrm{Pol} / \mathrm{Nec}$ & May & 25 & Vegetative \\
\hline Gicha & Cyperus spp. & Cyperaceae & 1.92 & Grass & Pollen & August & 5 & Seed/Veg \\
\hline Mango & Mangifera indica & Anacardiaceae & 1.92 & Tree & $\mathrm{Pol} / \mathrm{Nec}$ & April & 25 & Seed \\
\hline Gumero & Capparis micrantha & Capparidaceae & 1.74 & Shrub & $\mathrm{Pol} / \mathrm{Nec}$ & June & 25 & Seed/Veg \\
\hline Chiret & Agave sisalana & Agavaceae & 1.59 & Shrub & $\mathrm{Pol} / \mathrm{Nec}$ & September & 30 & Vegetative \\
\hline $\begin{array}{l}\text { Qundo } \\
\text { Berberie }\end{array}$ & Schinus molle & Anacardiaceae & 1.39 & Tree & $\mathrm{Pol} / \mathrm{Nec}$ & April & 30 & Seed \\
\hline Aluma & Achyranthus aspera & Amaranthaceae & 1.25 & Herb & $\mathrm{Pol} / \mathrm{Nec}$ & September & 10 & Seed \\
\hline Teketila & Tapinanthus aurantias & Loranthaceae & 1.22 & Shrub & $\mathrm{Pol} / \mathrm{Nec}$ & September & 25 & undefined \\
\hline Dokima & Syzygium guineens & Myrtaceae & 1.11 & Tree & $\mathrm{Pol} / \mathrm{Nec}$ & April & $45-75$ & Seed \\
\hline Suf & Helianthus annuus & Asteraceae & 1.09 & Herb & $\mathrm{Pol} / \mathrm{Nec}$ & October & 15 & Seed \\
\hline Ekima & Terminalia glaucescens & Combretaceae & 0.85 & Tree & $\mathrm{Pol} / \mathrm{Nec}$ & April & 15 & Seed \\
\hline
\end{tabular}

Table 13: Bee plant species of the study area identified from the trapped pollen analysis

\begin{tabular}{|c|c|c|c|}
\hline Scientific Name & Family Name & Pollen Weight (gm) & $\%$ \\
\hline Acacia tortolis & Mimosaceae & 32.39 & 7.74 \\
\hline Ocimum bacilicum & Lamiaceae & 29.23 & 6.99 \\
\hline Becium grandiflorum & Lamiaceae & 26.97 & 6.45 \\
\hline Hypoestes trifolia & Acanthaceae & 26.95 & 6.44 \\
\hline Sorghum bicolor & Poaceae & 25.53 & 6.10 \\
\hline Bidens spp. & Asteraceae & 24.46 & 5.85 \\
\hline Guizotia abyssinica & Asteraceae & 23.83 & 5.69 \\
\hline Echinops Spp. & Asteraceae & 17.87 & 4.27 \\
\hline Vernonia Spp. & Asteraceae & 14.81 & 3.54 \\
\hline Grewia bicolor & Tiliaceae & 13.81 & 3.30 \\
\hline Brassica spp. & Brassicaceae & 11.65 & 2.78 \\
\hline Eucalyptus camaldulensis & Myrtaceae & 11.46 & 2.74 \\
\hline Aloe berhana & Liliaceae & 11.25 & 2.69 \\
\hline Un-Identified spp. & Poaceae & 10.50 & 2.51 \\
\hline Acacia asak & Mimosaceae & 10.17 & 2.43 \\
\hline Ziziphus spinachristi & Rhamnaceae & 10.09 & 2.41 \\
\hline Opuntia Spp. & Cactaceae & 9.79 & 2.34 \\
\hline Acacia mellifera & Mimosaceae & 9.44 & 2.26 \\
\hline Euphorbia Spp. & Euphorbiaceae & 8.94 & 2.14 \\
\hline Acacia seyal & Mimosaceae & 8.05 & 1.92 \\
\hline Sesamum indicum & Pedaliaceae & 7.84 & 1.87 \\
\hline Vicia faba & Papilionaceae & 7.63 & 1.82 \\
\hline Pterolobium stellatum & Caesalpiniaceae & 7.31 & 1.75 \\
\hline Cordia africana & Boraginaceae & 6.70 & 1.60 \\
\hline Lepidium sativum & Brassicaceae & 4.65 & 1.11 \\
\hline Trifolium Spp. & Papilionaceae & 4.28 & 1.02 \\
\hline Plectranthus spp. & Lamiaceae & 3.76 & 0.90 \\
\hline Zantadescha spp. & Araceae & 3.75 & 0.90 \\
\hline Acacia brevispica & Mimosaceae & 3.52 & 0.84 \\
\hline Eluesine folicofolia & Poaceae & 3.38 & 0.81 \\
\hline Justicia spp. & Acanthaceae & 3.36 & 0.80 \\
\hline Schinus molle & Anacardiaceae & 3.29 & 0.79 \\
\hline Achyranthus aspera & Amaranthaceae & 2.92 & 0.70 \\
\hline Helianthus annuus & Asteraceae & 2.53 & 0.60 \\
\hline Rumex nervosus & Polygonaceae & 2.34 & 0.56 \\
\hline Capparis micrantha & Capparidaceae & 2.10 & 0.50 \\
\hline Cyperus spp. & Cyperaceae & 1.92 & 0.46 \\
\hline Mangifera indica & Anacardiaceae & 1.92 & 0.46 \\
\hline Balanite aegyptica & Balanitaceae & 1.77 & 0.42 \\
\hline Agave sisalana & Agavaceae & 1.59 & 0.38 \\
\hline
\end{tabular}


Floral phenology and pollen potential of honey bee plants in North-East dry land areas of Amhara...

\begin{tabular}{cccc} 
Zea mays & Poaceae & 1.34 & 0.32 \\
Tapinanthus aurantias & Loranthaceae & 1.22 & 0.29 \\
Syzygium guineens & Myrtaceae & 1.11 & 0.27 \\
Terminalia glaucescens & Combretaceae & 0.85 & 0.20 \\
Plantago spp. & Plantaginaceae & 0.18 & 0.04 \\
\hline
\end{tabular}

Table 14: Identified bee plant families from trapped pollen analysis

\begin{tabular}{|c|c|c|c|c|}
\hline No. & Family Name & $\begin{array}{c}\text { Pollen dry } \\
\text { Weight (gm) }\end{array}$ & $\%$ & No. of Plant Spp. Included \\
\hline 1 & Asteraceae & 83.50 & 19.95 & 5 \\
\hline 2 & Mimosaceae & 63.57 & 15.19 & 5 \\
\hline 3 & Lamiaceae & 59.96 & 14.33 & 3 \\
\hline 4 & Poaceae & 40.75 & 9.74 & 4 \\
\hline 5 & Acanthaceae & 30.31 & 7.24 & 2 \\
\hline 6 & Brassicaceae & 16.30 & 3.90 & 2 \\
\hline 7 & Tiliaceae & 13.81 & 3.30 & 1 \\
\hline 8 & Myrtaceae & 12.57 & 3.00 & 2 \\
\hline 9 & Papilionaceae & 11.91 & 2.85 & 2 \\
\hline 10 & Liliaceae & 11.25 & 2.69 & 1 \\
\hline 11 & Rhamnaceae & 10.09 & 2.41 & 1 \\
\hline 12 & Cactaceae & 9.79 & 2.34 & 1 \\
\hline 13 & Euphorbiaceae & 8.94 & 2.14 & 1 \\
\hline 14 & Pedaliaceae & 7.84 & 1.87 & 1 \\
\hline 15 & Caesalpiniaceae & 7.31 & 1.75 & 1 \\
\hline 16 & Boraginaceae & 6.7 & 1.60 & 1 \\
\hline 17 & Anacardiaceae & 5.21 & 1.25 & 2 \\
\hline 18 & Araceae & 3.75 & 0.90 & 1 \\
\hline 19 & Amaranthaceae & 2.92 & 0.70 & 1 \\
\hline 20 & Polygonaceae & 2.34 & 0.56 & 1 \\
\hline 21 & Capparidaceae & 2.1 & 0.50 & 1 \\
\hline 22 & Cyperaceae & 1.92 & 0.46 & 1 \\
\hline 23 & Balanitaceae & 1.77 & 0.42 & 1 \\
\hline 24 & Agavaceae & 1.59 & 0.38 & 1 \\
\hline 25 & Loranthaceae & 1.22 & 0.29 & 1 \\
\hline 26 & Combretaceae & 0.85 & 0.20 & 1 \\
\hline 27 & Plantaginaceae & 0.18 & 0.04 & 1 \\
\hline
\end{tabular}

In general, 31 families comprising 59 species of bee plants were identified in the study area from the survey, inventory and trapped pollen analyses. About $27.12 \%$ of the identified plants were herbaceous species while $33.9 \%$ and $27.12 \%$ were shrubs and trees respectively. The rest proportion of these plants was grass species (Table $15)$.

Table 15: Pollen collection by life form of the honey plants identified in the wereda

\begin{tabular}{ccccc} 
Life Form & No. of Species & \% & Pollen Weight & \% \\
\hline Grasses & 7 & 11.86 & 15.98 & 3.82 \\
Herbs & 16 & 27.12 & 151.12 & 36.11 \\
Shrubs & 20 & 33.90 & 163.55 & 39.08 \\
Trees & 16 & 27.12 & 87.8 & 20.98 \\
\hline Total & $\mathbf{5 9}$ & $\mathbf{1 0 0 . 0 0}$ & $\mathbf{4 1 8 . 4 5}$ & $\mathbf{1 0 0 . 0 0}$ \\
\hline
\end{tabular}

As a result, a total of 418.45 gm of pollen was collected by honey bees during the study period from herbaceous species $(36.11 \%)$, shrubs $(39.08 \%)$ and trees $(20.98 \%)$ which further explained that the value or the contribution of honey plant species was not mainly depending on the number of species and the abundance and density of the plant species are the main determinant factors. Generally, the total pollen collected by bees from the different life forms was in agreement with the result obtained from sample quadrant plot analysis in this study. Moreover, the numbers of shrub plant species identified in trapped pollen were accounting $39.53 \%$ of the inventoried plant species while $25.58 \%$ and $20.93 \%$ of the plant species in trapped pollen were trees and herbaceous plant species respectively and the rest the grass species.

Regarding pollen collection by flowering months, because of the reason that flowering period differs with different agro-ecologies based on their moisture contents, the highest volume of pollen (74.64\%) was collected during the main rainy season (August through October) with peak in September (37.58\%). Among flowering periods 
Floral phenology and pollen potential of honey bee plants in North-East dry land areas of Amhara...

of the year, the lowest amount of pollen was collected in November (2.06\%) and May (3.83\%) (Table 16). The daily mean pollen collection was $2.32 \pm 0.0378 \mathrm{gm}$ while the maximum was $5.34 \mathrm{gm}$ throughout the study period.

Table 16: Pollen collection by months during the study period

\begin{tabular}{ccc}
\hline Month & Pollen Weight & $\%$ \\
\hline September & 157.24 & 37.58 \\
October & 68.13 & 16.28 \\
November & 8.62 & 2.06 \\
May & 16.04 & 3.83 \\
June & 49.04 & 11.72 \\
July & 40.81 & 9.75 \\
August & 78.57 & 18.78 \\
\hline
\end{tabular}

The number of plant species included in a family for the computation of pollen dry weight by plant families identified has showed a significant difference $(\mathrm{P} \approx 0.01)$. Consequently, Asteraceae, Mimosaceae and Lamiaceae were the major pollen source families in the study area contributed $19.95 \%, 15.19 \%$ and $14.33 \%$ of the total pollen collected respectively while Plantaginaceae was the least pollen contributor $(0.04 \%)$.

\section{Conclusion}

The study has confirmed that criterion set by respondents in prioritizing honey bee plants was in agreement with the amount of pollen collection. Thus, very good plants in each of the criterion have provided the highest pollen for the bees as a food.

The appropriate implementation of mellissopalynological methods have confirmed that the local floras are reflected in the corresponding spectrum of pollen types represented in the collected pollen pellets. This further explained the importance of apiculture for vegetation characterization and identification of geographical origins. Of course, a final perspective could be obtained by considering the relative breadth and depth of plant species utilization by honey bees.

Although a narrow group of species in a wide spectrum of floral choices is truly used at significant levels, fewer number of flowering plant species out of the numerous flora of the country were visited by the honey bees in the study area.

Consequently, based on the techniques that we have employed, Acacia tortolis, Ocimum bacilicum, Becium grandiflorum, Hypoestes trifolia, Sorghum bicolor, Bidens spp., Guizotia abyssinica, Echinops Spp., Vernonia Spp., Grewia bicolor, Brassica spp., Eucalyptus camaldlensis, Aloe berhana, Un-Identified spp., Acacia asak, Ziziphus spinacristi, Opuntia Spp., Acacia mellifera, Euphorbia Spp. and Acacia seyal have been found to be the dominant (major) honey bee plants of the study area. However, as compared to honey production potential of the area, only few numbers of pollen source plant species were identified due to time constraint.

Furthermore, the strong relationships between pollen yields collected from abundant bee plant species have essentially disproved the hypothesis stating that the availability of bee flora and beekeeping are independent in the study area. This in turn has led us to conclude that beekeeping productivity is strictly dependent on the availability of floral resources in the study area. Thus, in-depth analysis of bee plants throughout the year, determination of total carrying capacity, propagation, wise use and conservation of floral vegetation shall take a considerable attention for better integration of the sector with phenological flow and potential of flowering plants in the area. In this regard, the community should be supported with different conservation programs running in the area for better achievements. Furthermore, as most honey bee plants investigated in the study area are propagated by their seeds; collection, management and conservation of plant seeds should not be a sidelined activity.

\section{Acknowledgments}

We would like to acknowledge Mekelle University and Amhara regional agricultural research institute for allowing us to conduct this study and their valuable supports during experimentation. We are also pleased to acknowledge Holleta bee research and Sekota dry land agricultural research centers with their staffs for supporting the laboratory analysis and field works.

\section{References}

[1]. Adefress T, Agajjie T., Aster Y., Birhane K., Derege G., Elias Z., Mengistu A., and Worku A.. 2000. 'Participatory Farming System Characterization and Interventions Options for Sekota Woreda, Wag Himra Zone, Amhara Region' Working Paper, HARC, Ethiopia

[2]. Admasu Adi and Nuru Adgaba. 1999. Effect of honey bee pollination on seed yield and oil content of Noug. In proceedings of first National Conference of the Ethiopian Beekeepers Association, pp 67 - 73, Addis Ababa, Ethiopia 
[3]. Admasu Addi, Debissa Lemessa and Amssalu Bezabeh. 2004. Botanical inventory and phenology of bee plants in rift valley regions of East Showa Zone: Proceedings of the 13th annual conference of the Ethiopian Society of Animal Production (ESAP) August 25-27, 2004, Addis Ababa, Ethiopia,

[4]. AMAREW, 2006. Revised Annual Work Plan for 2006, Bahir Dar, Ethiopia

[5]. Amha Sebsebe. 2003. Practical aspect of beekeeping in Amhara region. Project proposal

[6]. Amssalu Bezabeh. 1999. Identification of major pollen source honey plants around Holleta Bee Research Centre. Proceedings of $7^{\text {th }}$ National Conference of Ethiopian Society of Animal Production

[7]. Amssalu, Bezabeh. 2004. Nature and phenology of honey plants in central highlands of Ethiopia, Proceedings of $12^{\text {th }}$ National Annual Conference of Ethiopian Society of Animal production

[8]. Amssalu, Bezabeh. 2007. Seasonal intensity of flowering and pollen-forage selectivity by honeybees, Apis melifera bandasii in central highlands of Ethiopia, Holleta Bee Research Centre, Ethiopia, ESAP

[9]. Araújo, P.A. 2005. Diversity of bees and their floral resources at altitudinal areas in the Southern Espinhaço Range, Minas Gerais, Brazil

[10]. CSAE. 2005. Census Report, Addis Ababa, Ethiopia.

[11]. EARO. 2001. Background on Beekeeping in the Ethiopian Context, Ethiopian Agricultural Research Organization, Apiculture Research Program, Research Strategy, Addis Ababa, Ethiopia

[12]. FAO. 1987. Beekeeping in Asia. FAO Agric. Service Bulletin no. 68/4, 112 pp.

[13]. Fichtl R and Admassu A. 1994. Honey Bee Flora of Ethiopia, Margraf Verlag, Weikersheim, Germany

[14]. Francis G. Smith. 1990. Beekeeping in the Tropics, Longmans, Tanganika, 6:62-77.

[15]. ILRI. 2000. 'Land Degradation and Strategies for Sustainable Development in the Ethiopian Highlands: Amhara Region,' SocioEconomics and Policy Research Working Paper 32, Nairobi, Kenya.

[16]. Lakew Desta, Menale Kassie, Benin S. and Pender J. 2000. Land Degradation and Strategies for Sustainable Development in the Ethiopian Highlands: Amhara Region. Socio - Economics and Policy Research working paper 32. ILRI, Nairobi, Kenya. pp 122

[17]. MoA. 1998. 'Agro-ecological zones of Ethiopia', National Resource management and regulatory Department, MOA, Working paper

[18]. Pearson W. D. and Braiden V. 1990. Seasonal Pollen Collection by Honeybees from Grass/Shrub Highlands in Canterbury, New Zealand, Journal of Apicultural Research 29 (4): 206 - 213

[19]. Segeren P. 2004. Beekeeping in the Tropics, Agromisa Foundation, Digigrafi, Wageningen, the Netherlands

[20]. SWoA. 2006. Annual Report. Sekota, Ethiopia

[21]. Tilahun Amede. 2003. Natural Resources Degradation and Environmental Concerns in the Amhara National Regional State: Impact on Food Security, Proceedings of the Natural Resource Management Conference held at Bahir Dar, Ethiopia, pp. 33-50. 